\title{
Optical instrumentation for chromospheric monitoring during solar cycle 25 at Paris and Côte d'Azur observatories
}

\author{
Jean-Marie Malherbe ${ }^{1, *}$, Thierry Corbard ${ }^{2}$, and Kevin Dalmasse ${ }^{3}$ \\ ${ }^{1}$ LESIA, Observatoire de Paris, PSL Research University, CNRS, 92195 Meudon, France \\ ${ }^{2}$ Université Côte d'Azur, Observatoire de la Côte d'Azur, CNRS, Laboratoire Lagrange, 06304 Nice, France \\ ${ }^{3}$ IRAP, Université de Toulouse, CNRS, CNES, UPS, 31028 Toulouse, France
}

Received 8 April 2020 / Accepted 21 June 2020

\begin{abstract}
We present the observing program proposed by Paris and Côte d'Azur Observatories for monitoring solar activity during the upcoming cycle 25 and providing near real time images and movies of the chromosphere for space-weather research and applications. Two optical instruments are fully dedicated to this task and we summarize their capabilities. Short-term and fast-cadence observations of the chromosphere will be performed automatically at Calern observatory (Côte d'Azur), where dynamic events, as flare development, Moreton waves, filament instabilities and Coronal Mass Ejections onset, will be tracked. This new set of telescopes will operate in 2021 with narrow bandpass filters selecting $\mathrm{H} \alpha$ and CaII K lines. We present the instrumental design and a simulation of future images. At Meudon, the Spectroheliograph is well adapted to the long-term and low-cadence survey of chromospheric activity by recently improved and optimized spectroscopic means. Surface scans deliver daily $(x, y, \lambda)$ datacubes of $\mathrm{H} \alpha$, CaII $\mathrm{K}$ and CaII $\mathrm{H}$ line profiles. We describe the nature of available data and emphasize the new calibration method of spectra.
\end{abstract}

Keywords: Sun / chromosphere / optical observations / solar cycle 25

\section{Introduction}

Solar activity is the primary driver of space weather and space climate (e.g., Schrijver et al., 2012; Pomoell \& Poedts, 2018). It varies over a wide range of time scales from several minutes with solar flares and Coronal Mass Ejections (CMEs; e.g., Shibata \& Magara, 2011) to hundreds of years with the $\approx 100$-year modulation (Gleissberg cycle) of the 11-year solar cycle (e.g., Hathaway, 2015). Both short-term and long-term full-disk observations of the Sun are thus critical for improving our understanding of solar activity at all time scales, but also for monitoring and predicting it in space weather forecast applications (e.g., Ueno et al., 2010; Malherbe et al., 2019).

Ground-based observations of the chromosphere are of particular interest, because this layer is the source of solar activity. Two international networks are already operating with several ground-based stations at various longitudes: the Global $\mathrm{H} \alpha$ Network (GHN, http://ghn.njit.edu) and the GONG $\mathrm{H} \alpha$ network (http://halpha.nso.edu). Counterparts in the hot corona above are observed in EUV by Solar Dynamics Observatory (SDO/AIA, NASA, since 2010) or radiotelescopes (e.g., Nançay, Nobeyama). Events at large distance of the Sun (up to $30 \mathrm{rad}$ ) are tracked by several wide angle, white light space

\footnotetext{
*Corresponding author: jean-marie.malherbe@obspm. fr
}

coronographs (SoHO/LASCO, ESA/NASA, since 1996; STEREO/NASA since 2006). Parker Solar Probe (NASA, launched in 2018) observes in situ the far corona and the Solar Orbiter mission (ESA) started early 2020. SDO/HMI provides in addition photospheric magnetograms and dopplergrams.

We summarize below the interest of chromospheric observations in the scope of space weather research and applications. Then we review the state of instrumentation dedicated to this task at Paris (OP) and Côte d'Azur (OCA) observatories in the context of the upcoming solar cycle 25. Section 2 presents new instrumentation for short-term, high-cadence monitoring of solar activity. The widely open data will be used by the french Air Force for space weather forecast and by the scientific community to investigate unstable phenomena as flares, Moreton waves or CMEs. Section 3 describes the long-term, low-cadence Spectroheliograph while Section 4 develops the wavelength calibration of new datacubes.

\subsection{Short-term, high-cadence chromospheric observations}

High-cadence $\mathrm{H} \alpha$ observations are widely used in the context of solar flare/CME analyses and monitoring (e.g., Deng et al., 2002; Huang et al., 2014). In the case of filament 
eruptions, such data allow one to follow the pre-, and early-, eruptive dynamics of the filament, which provide insights into both the CME triggering and driving mechanisms when combined with the analysis of other datasets such as photospheric magnetic field data (e.g., Joshi et al., 2014; Chen et al., 2018). Adopting the framework of the Torus Instability (one of the main models considered for CME trigger/driver; Kliem \& Török, 2006; Démoulin \& Aulanier, 2010), then H $\alpha$ imaging can further be used to monitor the slow rise of the filament and predict when it will reach the critical height for the onset of the instability (e.g., Filippov \& Zagnetko, 2008; McCauley et al., 2015).

More generally, high-cadence $\mathrm{H} \alpha$ observations are further interesting because they also register the chromospheric response to solar flares/CMEs, as flare ribbons (e.g., Wang et al., 2003; Jiang et al., 2014) and Moreton waves (e.g., Narukage et al., 2008; Muhr et al., 2010). Flare ribbons are "surface" brightenings (often observed in EUV and $\mathrm{H} \alpha$ ) caused by the interaction of energetic particles and thermal energy (emitted at the coronal reconnection region) with the lower and denser layers of the solar atmosphere (e.g., Schmieder et al., 1987; Fletcher et al., 2011). Moreton waves are large-scale disturbances that appear as arc-shaped, bright fronts in the center and blue wing of the $\mathrm{H} \alpha$ line (e.g., Moreton, 1960; Zhang et al., 2011) and which physical nature is still not yet fully understood (e.g., Liu et al., 2013; Warmuth, 2015). Both $\mathrm{H} \alpha$ flare ribbons and Moreton waves are chromospheric signatures of the coronal dynamics. Their spatial and temporal evolution thus provide crucial information on the flare/CME energetics (e.g., Gilbert et al., 2008; Veronig \& Polanec, 2015), which can be used to estimate the potential impact of the flare/CME on the Earth's magnetosphere in space weather forecast applications.

\subsection{Long-term, low-cadence chromospheric observations}

On the other hand, long-term, low-cadence full-disk observations of the chromosphere in $\mathrm{CaII} \mathrm{K}$ and $\mathrm{H} \alpha$ lines, such as the centennial Kodaikanal (Hasan et al., 2010) and Meudon collections (Malherbe \& Dalmasse, 2019), are gold mines for research on the solar cycle and its variability. The CaII K1v imaging (blue wing) allows us to derive the sunspots number and area (e.g., Bertello et al., 2016; Chatterjee et al., 2016), while the $\mathrm{H} \alpha$ imaging enables us to compute the number of filaments and their lengths (e.g., Mouradian \& Soru-Escaut, 1993; Hao et al., 2015). All these quantities are good tracers of solar activity that permit us to probe the properties of the solar cycle (amplitude, duration, activity level). The accumulation of such data series and products over very long periods is thus essential to study and improve our understanding of the solar cycle variability and modulation (including the Gleissberg modulation or the occurrence of grand minima such as the Maunder minimum; e.g., Clette et al., 2015; Webb et al., 2018), both of which directly affect space weather.

The study of rare events is yet another research field to which long-term, full-disk CaII $\mathrm{K} 1 v$ and $\mathrm{H} \alpha$ archives contribute. Determining the frequency of rare events such as large sunspot groups is key to both solar dynamo and climatology (e.g., Jiang et al., 2015; Schmieder, 2018). Recent work by Nagy et al. (2017) shows that the emergence of a large-scale bipolar active region into the solar atmosphere can have major effects on the properties of the solar cycle and its cycle-to-cycle variability. It can introduce an activity asymmetry and time lag between the North and South solar hemispheres (similar to the one already observed; e.g., McIntosh et al., 2013; Svalgaard \& Kamide, 2013), or trigger a grand minimum in the subsequent cycles (similar to the Maunder minimum; e.g., Spoerer \& Maunder, 1890; Ribes \& Nesme-Ribes, 1993).

CaII K3 (line center) and $\mathrm{H} \alpha$ imaging can also be used to probe the magnetic properties of solar active regions and the energy of solar flares in old archives, both of which are essential to research on extreme solar flares for the development of space weather prediction tools (e.g., Gopalswamy, 2017; Tsiftsi \& De la Luz, 2018). For instance, CaII K spectroheliograms provide a means to estimate the magnetic flux in plages and sunspots, thus enabling the construction of pseudomagnetograms (e.g., Harvey \& White, 1999; Pevtsov et al., 2016). Using empirical laws derived from observations and MHD models, one can further quantify the flare-energy release from $\mathrm{H} \alpha$ bright ribbons (e.g., Toriumi et al., 2017), and/or the maximum energy available for a solar flare from the CaII K size and area of an active region (e.g., Aulanier et al., 2013).

\section{MeteoSpace at Calern}

The MeteoSpace instrument will operate in 2021 at Calern (OCA, $1270 \mathrm{~m}$ elevation, the sunniest station in France). It is a fully automated system dedicated to short-term, high-cadence monitoring of chromospheric activity. For that purpose, three telescopes will be available (Malherbe et al., 2019): two for $\mathrm{H} \alpha$ line and one for $\mathrm{CaII} \mathrm{K}$ line.

MeteoSpace will complete the world-wide $\mathrm{H} \alpha$ survey with enhanced features. It will join the GHN network, composed of many heterogeneous instruments in terms of cadence, spatial resolution, seeing, filter type and bandwidth. In terms of capabilities, MeteoSpace is close to the homogeneous GONG $\mathrm{H} \alpha$ network (same filters), but it will offer improved temporal resolution (15 s) and two $\mathrm{H} \alpha$ channels (line center and blue wing). MeteoSpace will also propose CaII K systematic images, with faster cadence $(60 \mathrm{~s})$ than previous routines (such as PSPT); $\mathrm{CaII} \mathrm{K}$ is more chromospheric than the space-borne SDO/AIA 1600 and $1700 \AA$ channels.

The optical diameter of the three imagers is $100 \mathrm{~mm}$ with $985 \mathrm{~mm}$ equivalent focal length. The filters bandwidth, optical resolution on the Sun and observing cadence are given by Table 1. Instruments are enclosed in a temperature controlled box (active heating, passive cooling) supported by an equatorial mount. The detectors are QSI 660i and 690i air cooled cameras using shutterless interline CCD sensors from Sony (3000 dynamic range, 12 bits).

MeteoSpace is the first solar automatic station in France. All operations are fully robotized: weather checking, opening and closing the dome, catching the Sun, solar tracking, data acquisition, processing and transfer to the archive, and website update. MeteoSpace is supervised by a main computer connected to several sub-systems, for environment (weather station and full-sky camera), mount steering, instrument and camera control, data archiving and webservice. Two scintillometers (AiryLab company) provide an estimate of flux and seeing conditions ( $1^{\prime \prime}$ typical at Calern) for each image. The instrument 
Table 1. Capabilities of the MeteoSpace and Spectroheliograph instruments.

\begin{tabular}{|c|c|c|c|c|c|}
\hline Instrument & CWL & FWHM & Cadence & Simultaneous wavelengths & Spatial pixel \\
\hline Hydrogen & $6562.3 \AA$ & $0.46 \AA$ & $20 \mathrm{~s}$ & 1 & $0.96^{\prime \prime}$ \\
\hline CaIIK & $3933.7 \AA$ & $1.50 \AA$ & $60 \mathrm{~s}$ & 1 & $0.78^{\prime \prime}$ \\
\hline \multicolumn{6}{|c|}{ Spectro heliograph } \\
\hline Hydrogen & $6562.8 \AA$ & $0.155 \AA$ & 2 datacubes/day & $>20$ & $1.1^{\prime \prime}$ \\
\hline CaIIK & $3933.7 \AA$ & $0.093 \AA$ & 2 datacubes/day & $>30$ & $1.1^{\prime \prime}$ \\
\hline
\end{tabular}

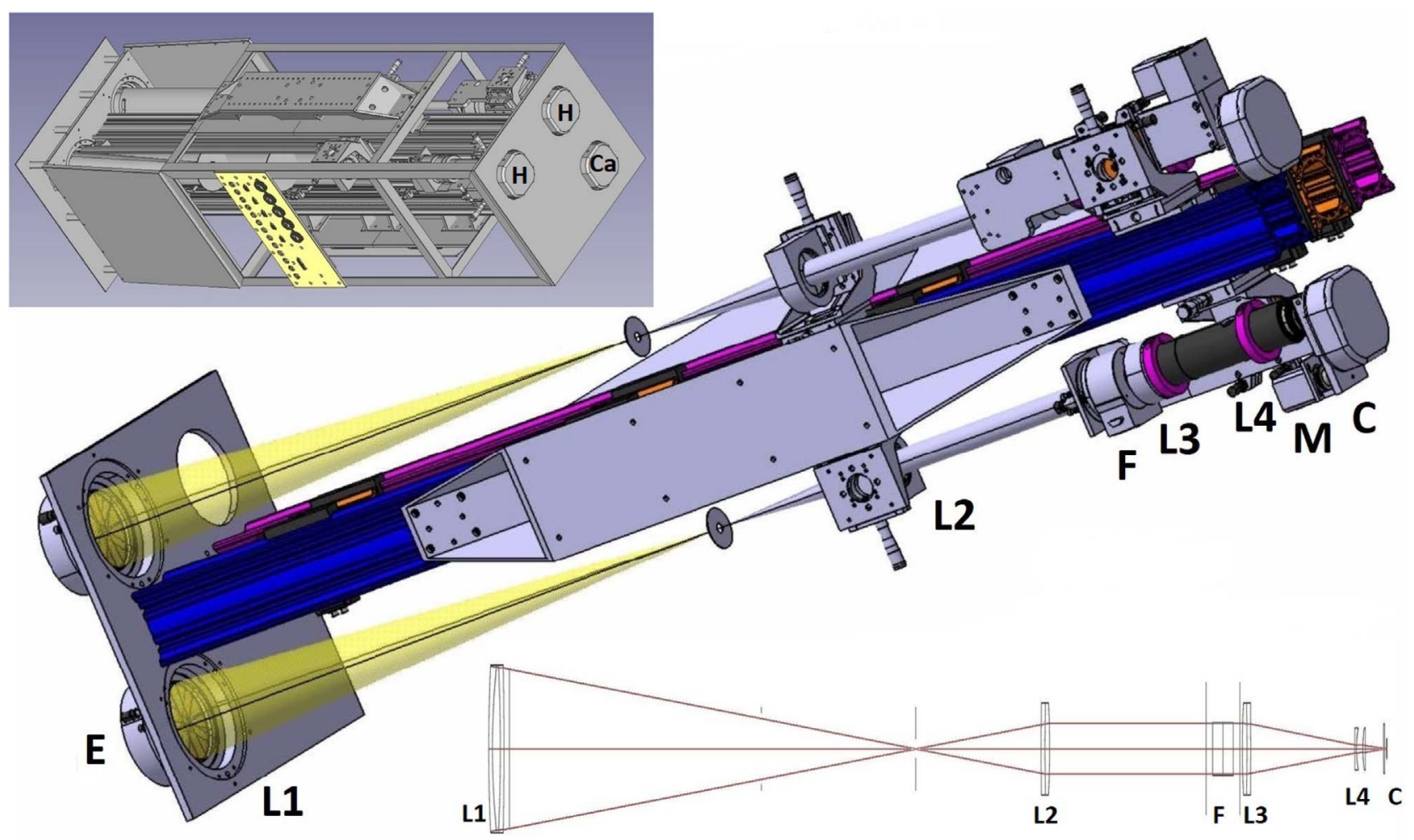

Fig. 1. Top: the MeteoSpace overall structure $(0.5 \times 0.5 \times 1.7 \mathrm{~m})$ enclosing three instruments; $\mathrm{H}=\mathrm{H} \alpha$; $\mathrm{Ca}=\mathrm{CaII} \mathrm{K}$. Middle: details of the two $\mathrm{H} \alpha$ telescopes; $\mathrm{E}=$ energy rejection filter (Baader); L1 = entrance objective (Takahashi TSA102); L2/L3 = afocal magnifying system; $\mathrm{F}$ = DayStar Quantum PE Fabry Pérot filter; L4 = field corrector/focuser; $\mathrm{M}=$ focuser motor; $\mathrm{C}=$ cooled CCD camera (QSI 660i). Bottom: optical design of the $\mathrm{H} \alpha$ telescopes.

is protected by many house-keeping procedures, including weather phenomena; in case of failure, a short messaging service (SMS) generates an alert to the local staff.

Quick look data (JPEG) will be available in real time, while scientific images (FITS) will be delivered a few hours later after calibration and corrections of dark current, geometric distorsion and rotation to present solar north up. Data will be processed and stored at Nice (30 Tera bytes available) but accessed through the BASS2000 national database dedicated to groundbased observations (http://bass2000.obspm.fr); a real-time channel is under development to disseminate high-cadence $\mathrm{H} \alpha$ images for space weather applications.

Figure 1 displays the two $\mathrm{H} \alpha$ imagers. They are enclosed in a $1.7 \times 0.5 \times 0.5 \mathrm{~m}^{3}$ box at $27^{\circ} \mathrm{C}$ temperature (maximum value at Calern in summer), with active heating but passive cooling. The entrance objective (L1) is a Takahashi TSA102. Both incorporate an afocal magnifying system $(\mathrm{L} 2 / \mathrm{L} 3, \times 1.2)$ for the temperature regulated Fabry-Pérot filter working in the F/30 beam (DayStar company, Quantum Pro series). The filter (F) is located in the pupil plane of the chamber, which includes L3, the filter plus a motorized field corrector/focuser (L4, M, Fig. 2). Flares, filament eruptions and CMEs will be observed with the first $\mathrm{H} \alpha$, line centered filter. The second filter is blue wing shifted $(-0.5 \AA)$ and dedicated to the detection of fast Moreton waves by running differences of consecutive images, as explained by Malherbe et al. (2019). The wavelength transmission curves of both filters are displayed in Figure 3. They were computed from $\mathrm{H} \alpha$ line profiles observed with the $14 \mathrm{~m}$ 


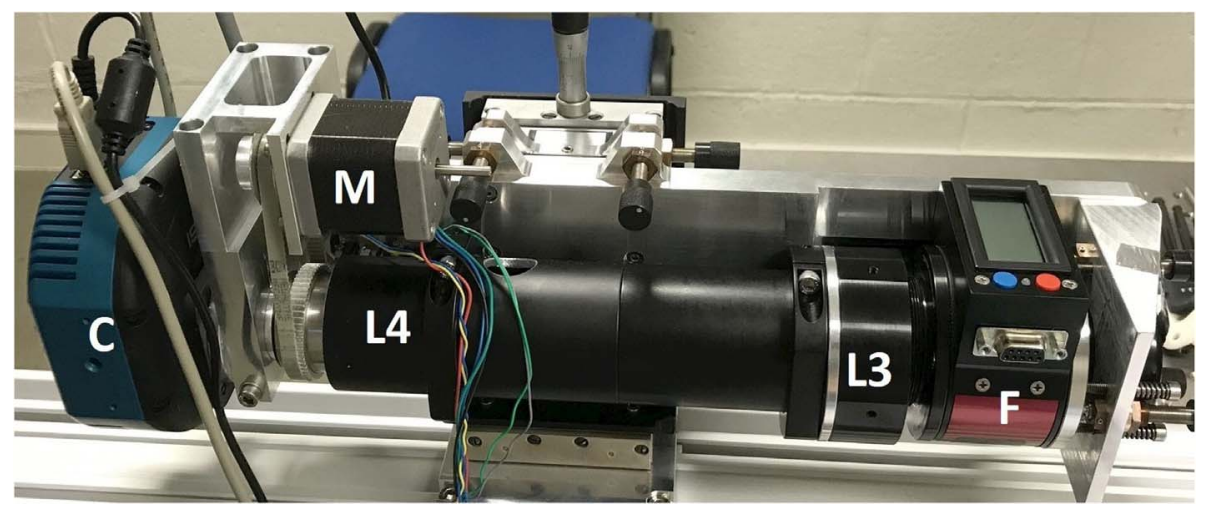

Fig. 2. The chamber of MeteoSpace $\mathrm{H} \alpha$ telescopes. $\mathrm{L} 3=$ chamber objective; $\mathrm{F}=$ DayStar Quantum PE Fabry Pérot filter; L4 = field corrector/focuser; $\mathrm{M}=$ focuser motor; $\mathrm{C}=$ cooled $\mathrm{CCD}$ camera (QSI 660i).

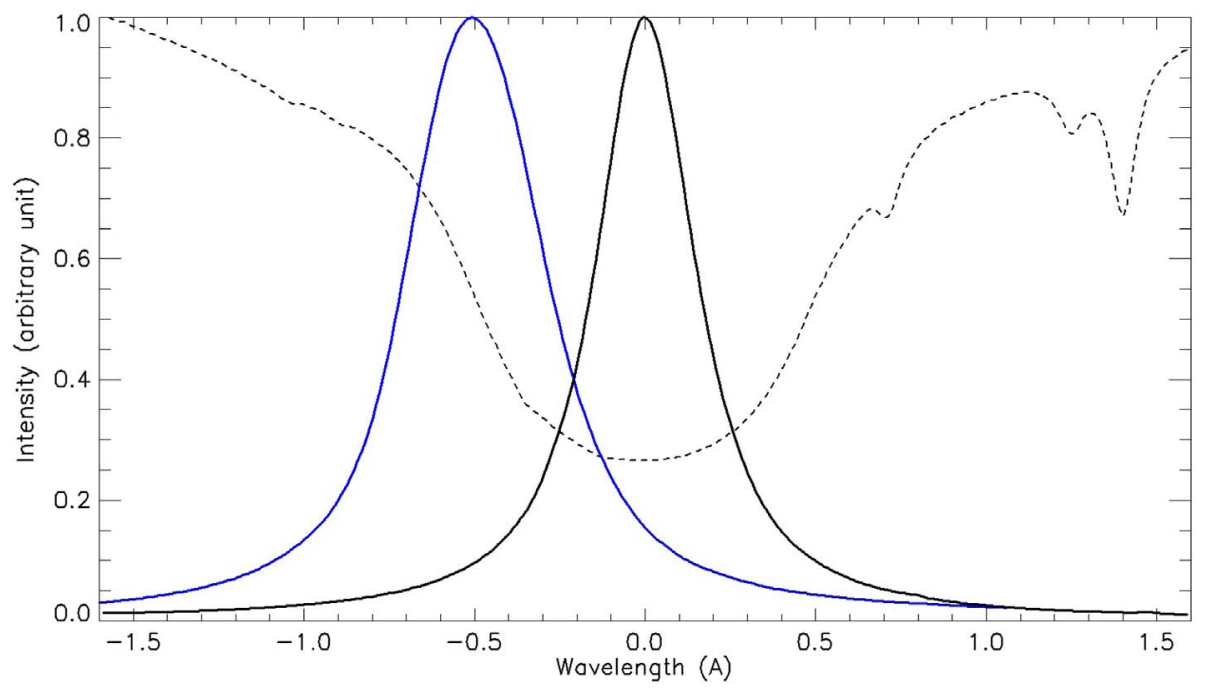

Fig. 3. The transmission curves of the line centered filter (black) and of the blue-shifted filter (blue) as a function of wavelength. The dashed line is the observed $\mathrm{H} \alpha$ line profile.

spectrograph (0.02 $\AA$ spectral resolution) of the Meudon Solar Tower. The curves derive from filter scans by the slit, after surface averaging, because the setup is a pupil plane application.

The CaII K filter has a bandwidth four times larger than Hydrogen filters and provides a proxy of chromospheric magnetic fields covering facular regions. The entrance objective of the telescope is a Takahashi FS102. The interference filter (from Barr Associates) is located in the image plane of the motorized magnifier/focuser $(\times 1.2$, not shown).

Figure 4 shows a simulation based on $(x, y, \lambda)$ dadacubes of the Meudon Spectroheliograph recorded on 13 Nov 2013 (near solar maximum) during a test campaign (Sect. 3). The two $\mathrm{H} \alpha$ images (core and blue wing centered) are obtained applying the wavelength transfer function of the respective filters (Malherbe et al., 2019) to line profiles. Then, both images can be combined in order to provide enhanced contrast pictures of filaments, plages and prominences at the limb. For that purpose, the blue wing is subtracted to the line core image after normalization by mean intensities measured in a large area around disk center. The CaII $\mathrm{K}$ image is obtained also by integration of the spectrum over the filter transmission curve. The result is intermediate between $\mathrm{K} 1 v$ and $\mathrm{K} 3$ spectroheliograms (Sect. 3); sunspots and bright active regions are well visible, but the filter bandpass make the filaments vanish.

The simulation allows to quantify the loss of structure contrast resulting from spectral integration over the Lorentzian transmission of filters in comparison with spectroscopic observations (Table 2). For $\mathrm{H} \alpha$, it reveals a loss for filaments and faculae. On the contrary there is a gain for sunspots, because the visibility of sunspots is better in the continuum and wings than in the line core. The same conclusions are amplified for $\mathrm{CaII} \mathrm{K}$, because the filter is broader. The visibility of prominences at the limb is not affected for $\mathrm{H} \alpha$ but is degraded in $\mathrm{CaII} \mathrm{K}$ for the same reason.

\section{Meudon Spectroheliograph}

Meudon Spectroheliograph operates daily since 1908 in CaII $\mathrm{K}$ and $\mathrm{H} \alpha$ chromospheric lines. It is not a filtergraph, but a spectrograph (Fig. 5) providing classical $(\lambda, x)$ spectra 


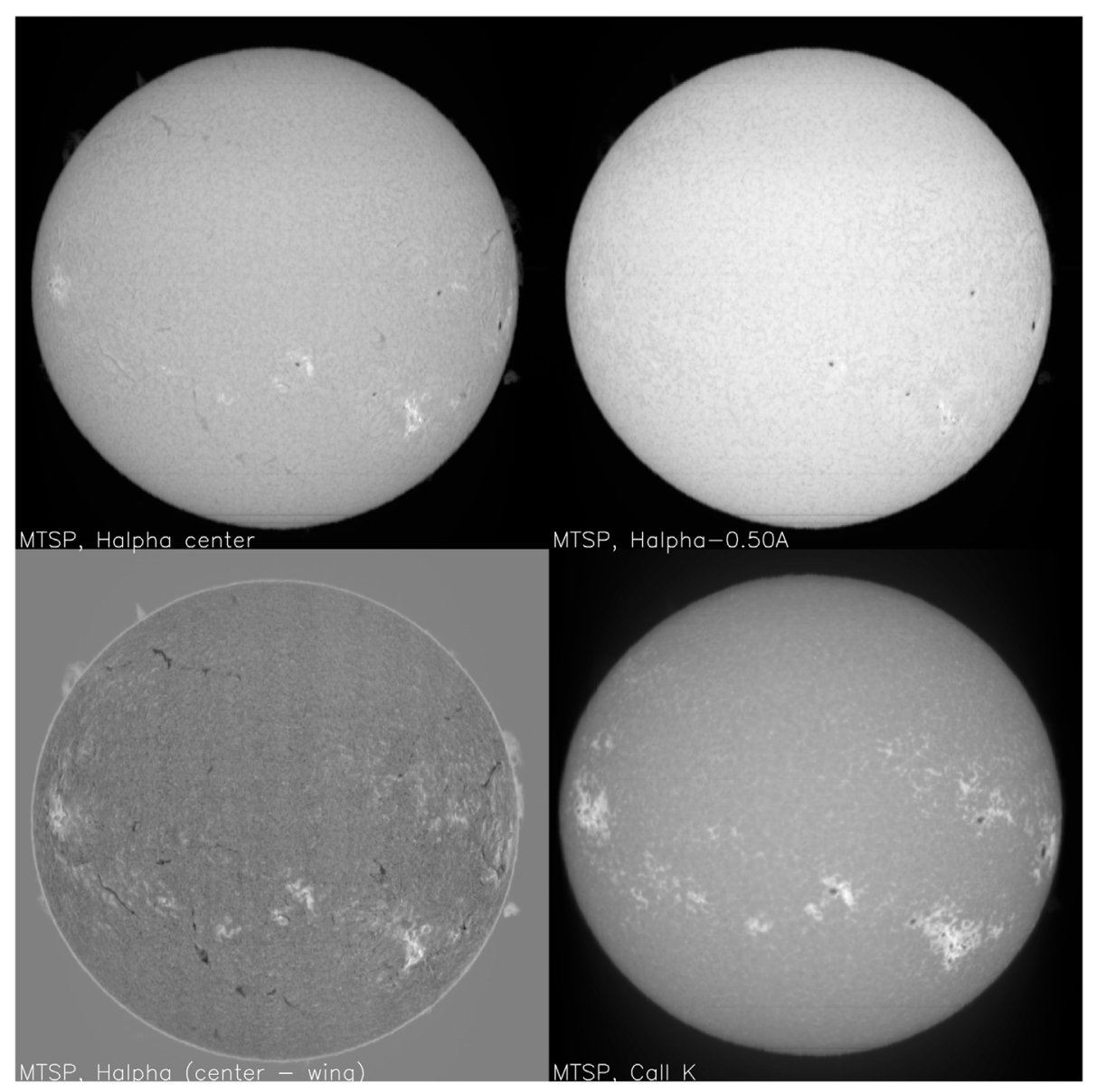

Fig. 4. Simulation of typical data that will be produced by the MeteoSpace instrument. Top (left to right): H $\alpha$ core and H $\alpha$ blue wing ( $-0.5 \AA$ ). Bottom (left to right): composite $\mathrm{H} \alpha$ image showing enhanced structures (line center minus blue wing) and CaII $\mathrm{K}$.

Table 2. Contrast loss of MeteoSpace structures compared to the Spectroheliograph at line center.

\begin{tabular}{lccc}
\hline $\begin{array}{l}\text { Solar } \\
\text { structure }\end{array}$ & $\begin{array}{c}\text { Hydrogen } \\
\text { filter 1 }\end{array}$ & $\begin{array}{c}\text { Hydrogen } \\
\text { filter 2 }\end{array}$ & $\begin{array}{c}\text { Calcium } \\
\text { filter }\end{array}$ \\
\hline Filaments & $-40 \%$ & $-47 \%$ & $-57 \%$ \\
Faculae & $-40 \%$ & $-49 \%$ & $-57 \%$ \\
Sunspots & $+16 \%$ & $+19 \%$ & $+100 \%$ \\
Prominences & $-5 \%$ & $-7 \%$ & $-44 \%$ \\
\hline
\end{tabular}

( $x$ abscissa along the slit). The second direction $y$, necessary to form solar images, comes from the scan of the solar surface. The main characteristics are reported in Table 1.

This instrument provided monochromatic images using glass and later film plates until 2001. The photographic device was replaced by a CCD array in 2002 (Malherbe \& Dalmasse, 2019). Since mid 2017, the detector is a fast SCMOS sensor which records full line profiles, so that $(x, y, \lambda)$ datacubes are registered in 3D FITS files, as shown by the online movie (see Supplementary Material, Movie S1 for details). Images are derived from the datacubes for precise wavelength positions along the line profiles.

The spectrograph is fixed and fed by a coelostat (two flat mirrors) and a $250 \mathrm{~mm}$ diameter objective $(\mathrm{O} 1,4 \mathrm{~m}$ focal length). The slit (S) of the spectrograph (30 microns or $1.5^{\prime \prime}$ width) is located in the solar image (37.2 mm diameter). The entrance objective (O1) is mounted on a motorized translator in order to scan the solar surface in $60 \mathrm{~s}$ typically.

The spectrograph has a $1.3 \mathrm{~m}$ focal length $(\mathrm{O} 2)$ and uses a 300 groves/mm grating $\left(17^{\circ} 27^{\prime}\right.$ blaze angle). Spectral lines form at interference orders $3(\mathrm{H} \alpha)$ or 5 (CaII K and $\mathrm{H})$. Orders are selected by filters (F). The spectral resolution is about $R=40,000$. The detector is a fast SCMOS sensor (PCO water cooled camera, 150,00 dynamic range, 14 bits) mounted on a SkyWatcher $0.4 \mathrm{~m}$ apochromatic objective (O3). The throughput is $100(\lambda, x)$ spectra/s. CaII $\mathrm{K}$ and $\mathrm{H}$ are always observed simultaneously (see Fig. 6 with solar atlas comparison). Long exposure observations are also done for prominences (5-10 times fainter) at the limb; in order to preserve the structures of the disk, an artificial "moon" (neutral density 0.9) is put against the solar image and is mechanically coupled to the objective translator O1.

Standard corrections include dark current, curvature of spectral lines and rotation (solar north up). Line profile datacubes (3D FITS) of $\mathrm{H} \alpha$, CaII $\mathrm{H}$ and $\mathrm{CaII} \mathrm{K}$, as well as classical images (JPEG and 2D FITS derived from datacubes at line center, wings or continuum) are freely available to the community through BASS2000 at http://bass2000.obspm.fr. Raw datacubes (TIF) are also stored online for specific use at ftp:// ftpbass2000.obspm.fr/pub/meudon/spc/tiff. Datacubes of $\mathrm{H} \beta$ line are scheduled for 2021. 


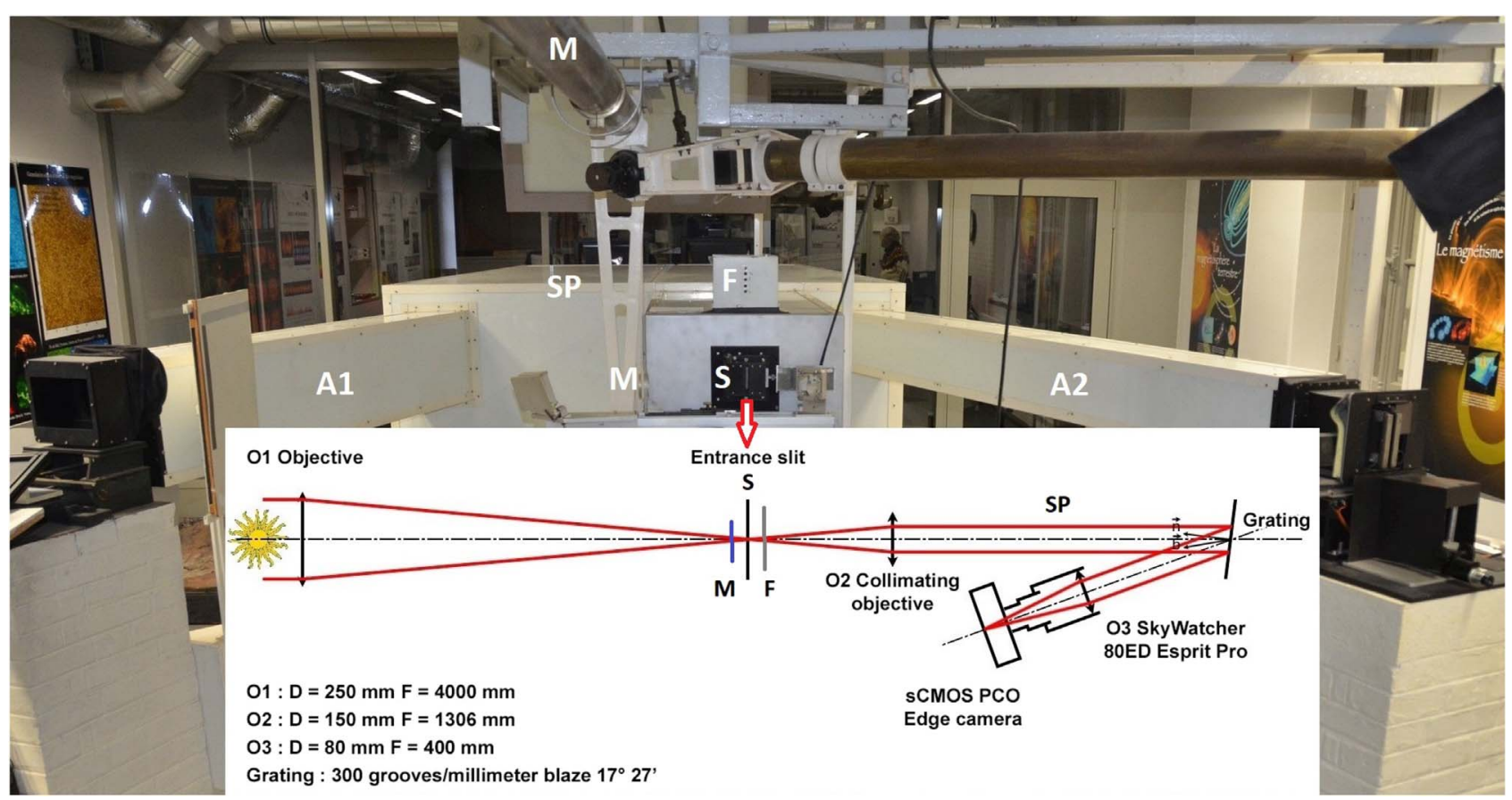

Fig. 5. The Spectroheliograph and optical scheme. $S=$ entrance slit; $S P=$ spectrograph; $F=$ interference filters; $M=$ neutral density "moon" translator for prominences; $\mathrm{O} 1=$ entrance objective and translator; $\mathrm{O} 2=$ collimator; $\mathrm{O} 3=$ chamber objective; $\mathrm{A} 1, \mathrm{~A} 2=$ historical "arms" with old photographic $13 \times 18 \mathrm{~cm}$ plate systems.

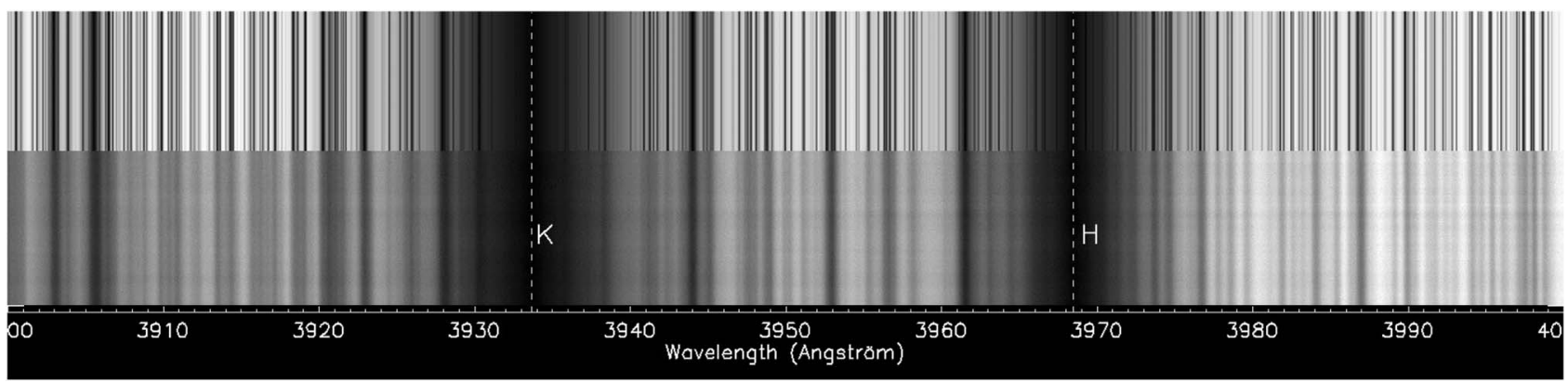

Fig. 6. Spectrum $(\lambda, x)$ between 3900 and $4000 \AA$ displaying CaII K and H lines near disk center. Top: high resolution atlas $\left(R=2 \times 10^{6}\right.$, Delbouille et al., 1973). Bottom: Spectroheliograph $(R=40,000)$.

In order to illustrate the Spectroheliograph capabilities, we chose datasets recorded near the last solar maximum (13 Nov 2013) with the new SCMOS detector (which was, at this moment, only available for tests). Figure 7 shows images extracted from $\mathrm{H} \alpha$ line profiles, with many sunspots, filaments and active regions. The composite image comes out from the difference between line core and the sum of the red and blue wings, after normalization by the average values computed in a quiet Sun region near disk center. This method makes all structures of the Sun appear together in a single frame (prominences, chromosphere at the limb, spots, filaments, faculae) with enhanced contrast. The Doppler velocity proxy is made of the contrast of line wings (ratio between the difference and sum of red and blue wings, after normalization by disk center values).

Figure 8 displays images derived from CaII $\mathrm{K}$ line profiles (CaII $\mathrm{H}$ line is also available, as observed simultaneously, and provides similar results). $\mathrm{K} 1 v$ and $\mathrm{K} 3$ are respectively the blue wing and line core. The composite image comes out from the difference between line center $(\mathrm{K} 3)$ and the sum of the red and blue wings $(\mathrm{K} 1 r+\mathrm{K} 1 v$ at $\pm 1.0 \AA)$, after normalization by the average values measured in a quiet region near disk center. As for $\mathrm{H} \alpha$, this method reveals all solar structures in a synthetic image (prominences, chromosphere at the limb, spots, filaments, bright plages) with better contrast.

\section{Wavelength calibration of Spectroheliograph datacubes}

Let us call $C[i, j, k]$ the datacubes provided by the Spectroheliograph FITS files with $i, j, k$ varying from 1 to respectively NAXIS1, NAXIS2 and NAXIS3 (pixel numbers). They need to 


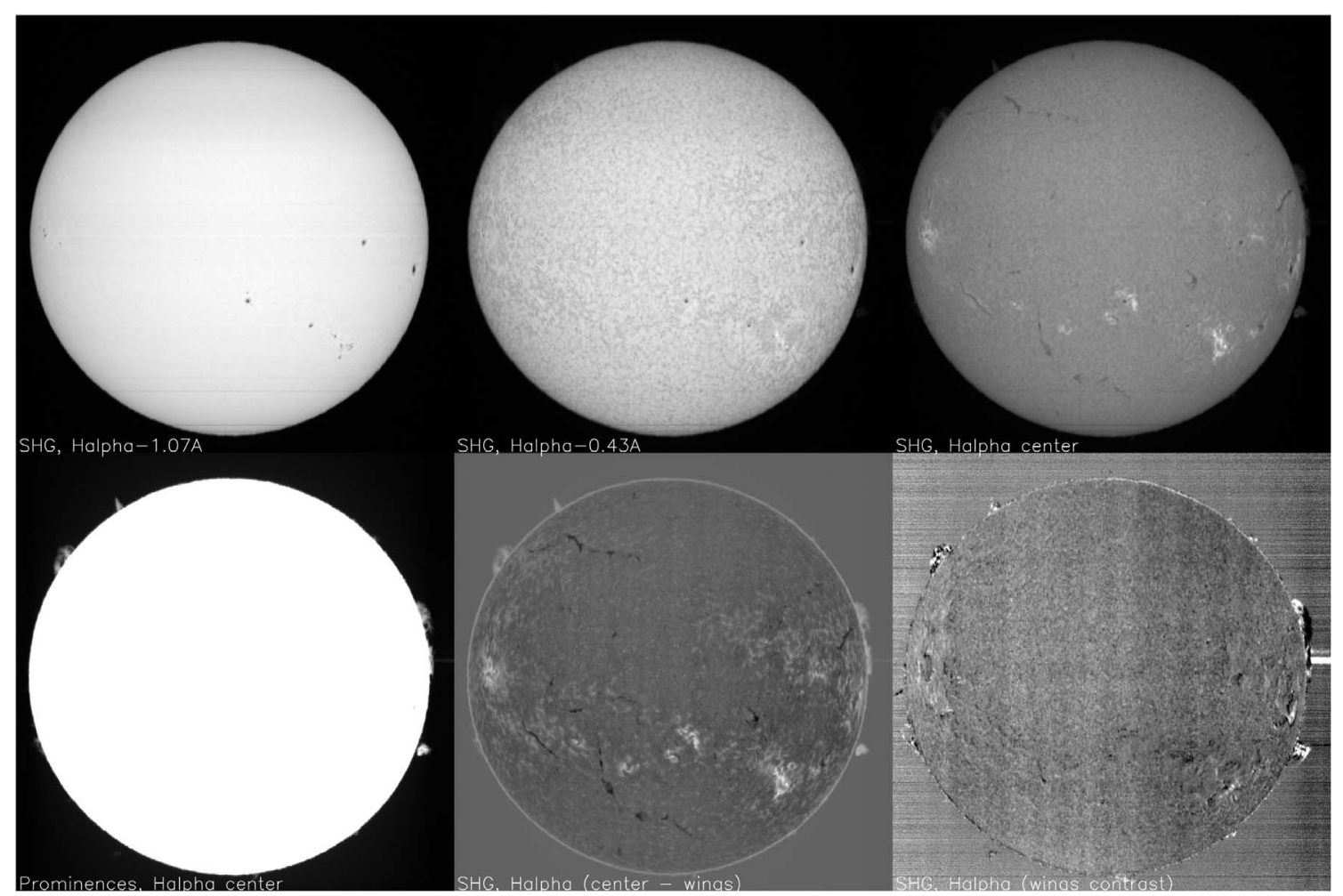

Fig. 7. Typical images issued from $\mathrm{H} \alpha$ datacubes produced by the Spectroheliograph. Top (left to right): continuum (-1.07 $\AA$ ), blue wing $(-0.43 \AA$ ) and line core. Bottom (left to right): limb prominences, composite image (line center minus red and blue wings) and Doppler proxy (contrast between both wings).

be calibrated along the wavelength axis $(k)$ in order to compute header CRPIX3 and CRVAL3 keywords (line center in pixel unit and in $\AA$, respectively, see Greisen \& Calabretta, 2002). They are roughly known in advance but can be precisely determined. We first locate the minimum of the integrated intensities of the cube images:

$$
\begin{gathered}
S_{\mathrm{obs}}[k]=\frac{\sum_{i, j} C[i, j, k]}{\mathrm{NAXIS} 1 \times \mathrm{NAXIS} 2} \quad k=1, \ldots, \text { NAXIS3 } \\
k_{\text {min }}=\text { index of } \min \left(S_{\mathrm{obs}}[k]\right) .
\end{gathered}
$$

The minimum of $S_{\text {obs }}$ at index $k_{\min }$ may not be strictly at the line core location $\lambda_{c}$ (keyword WAVELNTH). The idea is then to fit the observed line profile with a reference one $S_{\text {ref }}(\lambda)$ in order to identify precisely the location of the minimum and assign to it the value of $\lambda_{c}$. The reference spectrum is taken from the visible atlas by Delbouille et al. (1973) available online at the BASS2000 website (http://bass2000.obspm.fr) with a resolution of $\bar{\delta} \lambda=0.002 \AA$. The bandwidth of the cube is assumed unknown and therefore the reference spectrum is smoothed over a number of points $n$ that we also have to determine. We define the smoothed reference spectrum $\bar{S}_{\text {ref }}(\lambda)$ to which we want to compare the observed one by:

$$
\bar{S}_{\text {ref }}(\lambda)=\frac{1}{\delta \lambda} \int_{\lambda=\frac{\delta \lambda}{2}}^{\lambda+\frac{\delta \lambda}{2}} S_{\text {ref }}(\bar{\lambda}) \mathrm{d} \bar{\lambda} \quad \text { with } \quad \delta \lambda=n \bar{\delta} \lambda .
$$

The minimum value of this smoothed spectra is located at $\lambda_{s}(n)$ which is a function of $n$ and is slightly different from $\lambda_{c}$. The wavelengths corresponding to the different images of the cube are obtained by

$$
\lambda_{k}=\lambda_{s}(n)+\left(k-k_{\min }\right) \times \Delta \lambda
$$

where $\Delta \lambda$ (the wavelength increment in $\AA$ ) is given by the FITS keyword CDELT3:

$$
\Delta \lambda=\mathrm{CDELT} 3
$$

$$
= \begin{cases}0.0929 \AA & \text { for CaIIH }(0.0992 \AA \text { before } 2017 / 10 / 15) \\ 0.0932 \AA & \text { for CaIIK }(0.0995 \AA \text { before } 2017 / 10 / 15) \\ 0.1553 \AA & \text { for } \mathrm{H}_{\alpha}(0.1660 \AA \text { before } 2017 / 10 / 15)\end{cases}
$$

and $k_{\min }$ is an unknown fractional index. We assign $\lambda_{c}$ to the keyword CRVAL3 and compute CRPIX3 through:

$$
\text { CRPIX3 }=k_{\min }+\frac{\lambda_{\mathrm{c}}-\lambda_{s}(n)}{\Delta \lambda} .
$$

A linear scaling between the smoothed reference spectra and the observed one may not be always appropriate far from the line core. We allow a wavelength dependence of the linear term and write (see top panel of Fig. 9):

$$
\hat{S}_{\text {obs }}[k]=a+b\left(\lambda_{k}\right) \bar{S}_{\text {ref }}\left(\lambda_{k}\right) \quad k=1, \ldots, \text { NAXIS3 }
$$




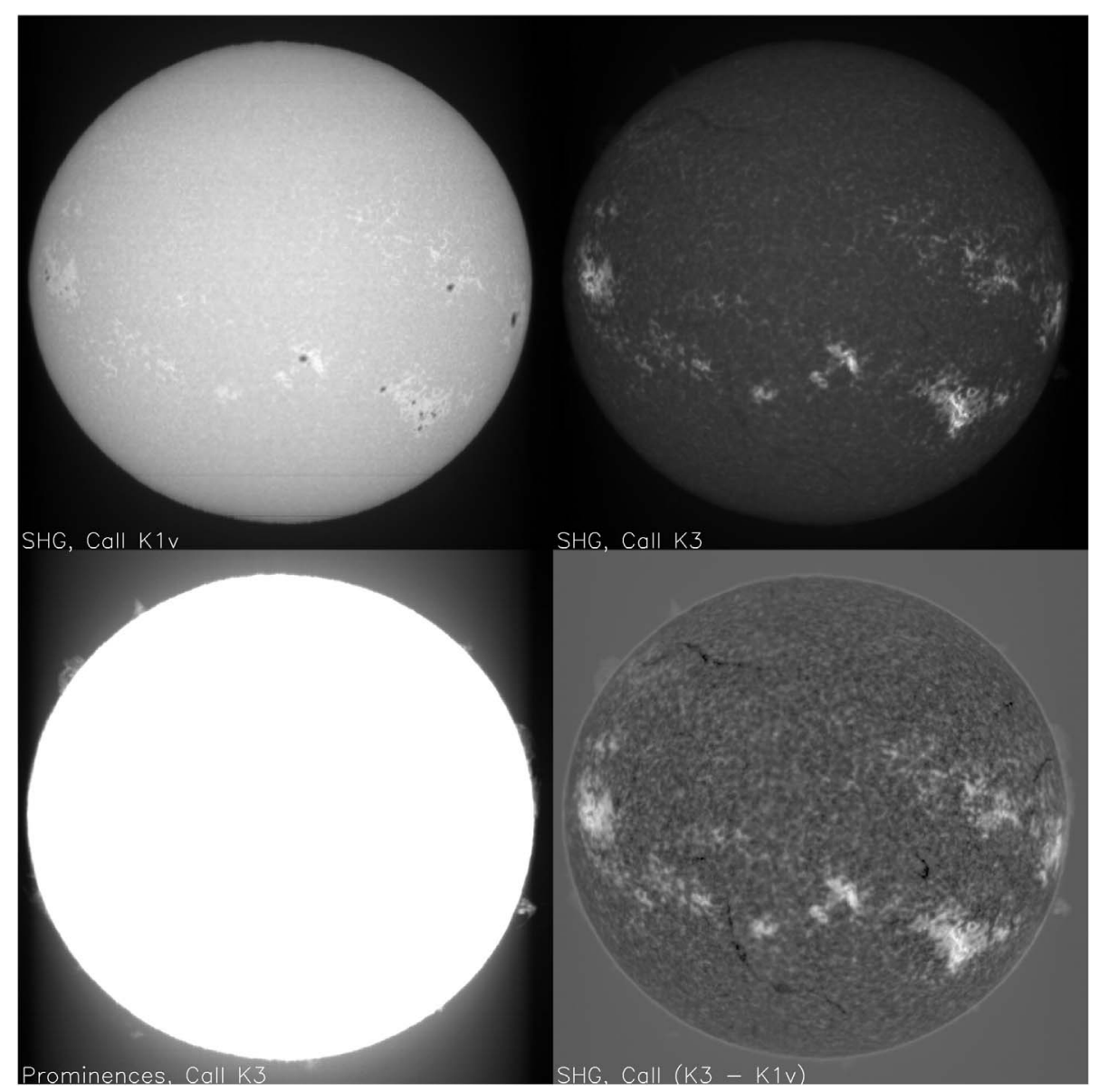

Fig. 8. Typical images issued from CaII K datacubes of the Spectroheliograph. Top (left to right): K1v (blue wing, -1.0 Å) and K3 (line core). Bottom (left to right): limb prominences (K3) and composite image (line center minus red and blue wings).

where:

$$
b\left(\lambda_{k}\right)=b+c\left(\lambda_{k}-\lambda_{\mathrm{c}}\right)+d\left(\lambda_{k}-\lambda_{\mathrm{c}}\right)^{2} .
$$

The six free parameters of the model are therefore: $a, b, c, d, n$ and $k_{\min }$. They could be obtained by minimizing the sum of squared residuals:

$$
\mathrm{SSR}=\sum_{k}\left(S_{\mathrm{obs}}[k]-\hat{S}_{\mathrm{obs}}[k]\right)^{2} .
$$

This quantity however will depend on the scaling parameters and will not permit an easy comparison for the goodness of the fit between different data cubes (quality index). We therefore prefer instead to build, from the observed profile, a profile that can always be compared to the reference one. From equation (5), we can define:

$$
\hat{S}_{\text {ref }}[k]=\frac{S_{\text {obs }}[k]-a}{b\left(\lambda_{k}\right)} .
$$

Furthermore, we use a weighting function chosen in order to give maximum weight to the points close to the line core:

$$
w[k]=\frac{1}{\left(\lambda_{k}-\lambda_{s}(n)\right)^{4}+1} .
$$

The six model parameters are thus obtained by minimizing a weighted sum of squared residuals (WSSR):

$$
\operatorname{WSSR}\left(a, b, c, d, n, k_{\min }\right)=\frac{\sum_{k} w[k]\left(\hat{S}_{\text {ref }}[k]-\bar{S}_{\text {ref }}\left(\lambda_{k}\right)\right)^{2}}{\sum_{k} w[k]} .
$$

We perform a multidimensional minimization using the NelderMead simplex algorithm (Gao \& Han, 2012). For the initial parameters we take $n=2 \Delta \lambda / \bar{\delta} \lambda, k_{\min }$ is obtained from equation (2) and, given these two values, $a, b, c, d$ are the least-squares solution to the linear matrix (5) which minimizes the SSR of equation (6).

For CaII K spectral line, Figure 9 shows the observed integrated intensities $S_{\text {obs }}$ compared to the scaled smoothed reference spectrum $\hat{S}_{\text {obs }}$ using the initial parameters (middle panel) and after optimization (bottom panel). Such calibrations are performed daily for $\mathrm{CaII} \mathrm{H}, \mathrm{K}$ and $\mathrm{H} \alpha$ lines; the result is available at ftp://solar-ftp.oca.eu/SHG/. 

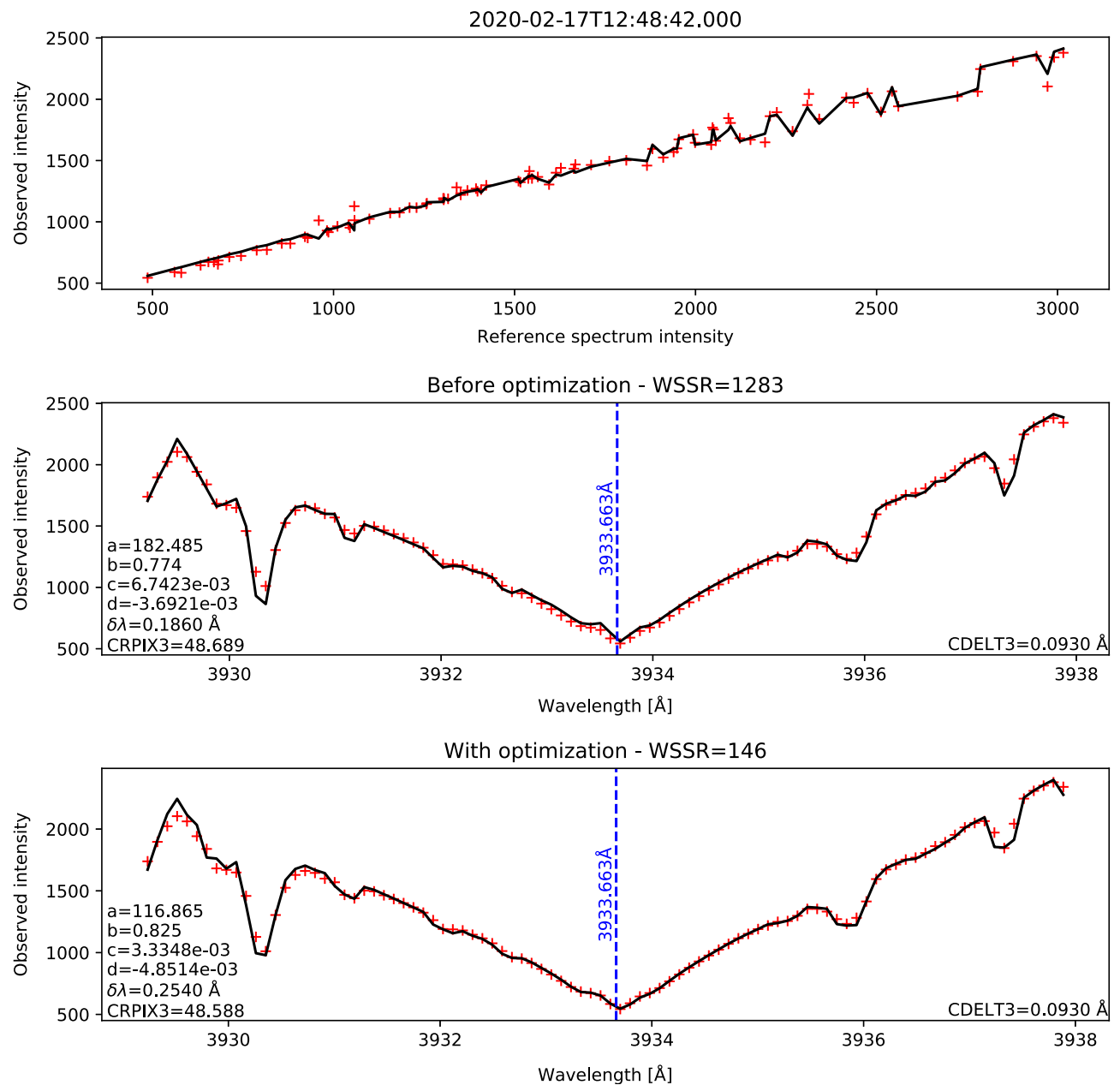

Fig. 9. Example of wavelength calibration of CaII K spectral line. The red crosses represent the observed disk-integrated intensities $S_{\text {obs }}$ (Eq. (1)). The black lines are the scaled smoothed reference spectrum $\hat{S}_{\text {obs }}$ (Eq. (5)). Top panel: $S_{\text {obs }}$ and $\hat{S}_{\text {obs }}$ are plotted as a function of the intensities of the smoothed reference spectrum $\bar{S}_{\text {ref }}$ (Eq. (3)). Middle and bottom panels: $S_{\text {obs }}$ and $\hat{S}_{\text {obs }}$ are plotted as a function of wavelength. The coefficients $a, b, c$ and $d$ of the fit are shown. The vertical blue line gives the line core position at $\lambda_{c}=3933.663 \AA$. The corresponding FITS index is given by CRPIX3. The interval over which the reference spectra is smoothed is given by $\delta \lambda$. WSSR is the resulting weighted sum of squared residuals. The top and middle panels give the fit with the initial parameters and the bottom panel gives the fit and parameters after optimization (lower WSSR).

\section{Conclusions}

New solar cycle 25 will be intensively observed at Paris and Côte d'Azur observatories with new and fast automatic instruments (MeteoSpace at Calern in 2021) or with improved capabilities existing ones (Meudon Spectroheliograph).

The MeteoSpace instrument is a new set of three automated telescopes using narrow Fabry-Pérot filters and dedicated to short-term, high-cadence $(20 \mathrm{~s})$ imagery of fast events such as flares, filament eruption, CME onset and Moreton waves. Simulations of $\mathrm{H} \alpha$ blue wing and line core images, as well as CaII $\mathrm{K}$ (magnetic proxy), are shown. In comparison with spectroscopic data, the Lorentzian shape of filters reduces the contrast of structures $(-40 \%$ on filaments and faculae in $\mathrm{H} \alpha$ ), but we suggest a simple method combining them to produce composite and enhanced images. Low cost pre-filters could also be added to limit the contrast loss to $-30 \%$. Capabilities could be extended at the end of the space-borne SDO mission by a fourth telescope for Stokes $\mathrm{V}$ measurements (magnetograms) in the blue wing of NaD1 $5896 \AA$ Aine.

The Spectroheliograph is dedicated to long-term, lowcadence observations of the chromosphere and now delivers $(x, y, \lambda)$ datacubes of $\mathrm{H} \alpha$, CaII $\mathrm{H}$ and $\mathrm{K}$ spectral lines, from which classical images are derived. A precise wavelength calibration method of datacubes, using comparison with atlas spectra, is proposed. Observation of $\mathrm{H} \beta$ line is the next extension.

All data are (or will be) freely available to the scientific community through a dedicated web service (BASS2000), which will offer an additional real-time channel to disseminate high cadence MeteoSpace images for space weather applications.

\section{Supplementary material}

Supplementary material is available at https://www.swscjournal.org/10.1051/swsc/2020032/olm 
Supplementary Movie S1: MPEG4 movie showing a typical wavelength exploration of Spectroheliograph datacubes (observation of 13 Nov 2013) in $\mathrm{H} \alpha$ and CaII $K$ lines. The current wavelength $(\AA)$ is indicated at the bottom of images. The mean line profile of disk center is superimposed with the moving wavelength index. Filaments appear only in the core of both lines.

Acknowledgements. We are indebted to the referees and to the editor for helpful comments and suggestions. We thank the observers operating the Spectroheliograph and the technical team of the MeteoSpace project. We acknowledge financial support of several institutions as DGA, CNRS/INSU (PNST), Paris and Côte d'Azur observatories, Ile de France DIM/ ACAV. This work has been supported through the UCA/JEDI investments managed by the National Research Agency (ANR) with the reference number ANR-15-IDEX-01. If you use data for publication, please insert the following sentence: "Spectroheliograph and MeteoSpace data are courtesy of Paris and Côte d'Azur observatories". The editor thanks two anonymous reviewers for their assistance in evaluating this paper.

\section{References}

Aulanier G, Démoulin P, Schrijver CJ, Janvier M, Pariat E, Schmieder B. 2013. The standard flare model in three dimensions. II. Upper limit on solar flare energy. $A \& A$ 549: A66. https://doi. org/10.1051/0004-6361/201220406.

Bertello L, Pevtsov AA, Tlatov A, Singh J. 2016. Solar Ca II K observations. Asian J Phys 25(3): 295-310.

Chatterjee S, Banerjee D, Ravindra B. 2016. A butterfly diagram and carrington maps for century-long CA II K spectroheliograms from the Kodaikanal observatory. Astrophys $J$ 827(1): 87. https://doi. org/10.3847/0004-637X/827/1/87.

Chen H, Duan Y, Yang J, Yang B, Dai J. 2018. Witnessing tethercutting reconnection at the onset of a partial eruption. Astrophys $J$ 869(1): 78. https://doi.org/10.3847/1538-4357/aaead1.

Clette F, Svalgaard L, Vaquero JM, Cliver EW. 2015. Revisiting the sunspot number. Sol Act Cycle 53: 35. https://doi.org/10.1007/9781-4939-2584-1_3.

Delbouille L, Roland G, Neven L. 1973. Atlas photometrique du spectre solaire de [lambda] 3000 a [lambda] 10000, Université de Liège.

Démoulin P, Aulanier G. 2010. Criteria for flux rope eruption: Nonequilibrium versus Torus instability. Astrophys J 718(2): 13881399. https://doi.org/10.1088/0004-637X/718/2/1388.

Deng Y, Lin Y, Schmieder B, Engvold O. 2002. Filament activation and magnetic reconnection. Sol Phys 209(1): 153-170. https://doi. org/10.1023/A:1020924406991.

Filippov B, Zagnetko A. 2008. Prominence height shows the proximity of an ejection. J Atmos Solr-Terr Phys 70(2-4): 614620. https://doi.org/10.1016/j.jastp.2007.08.035.

Fletcher L, Dennis BR, Hudson HS, Krucker S, Phillips K, et al. 2011. An observational overview of solar flares. Space Sci Rev 159 (1-4): 19-106. https://doi.org/10.1007/s11214-010-9701-8.

Gao F, Han L. 2012. Implementing the Nelder-Mead simplex algorithm with adaptive parameters. Comput Optim Appl 51(1): 259-277. https://doi.org/10.1007/s10589-010-9329-3.

Gilbert HR, Daou AG, Young D, Tripathi D, Alexander D. 2008. The Filament-Moreton wave interaction of 2006 December 6. Astrophys J 685(1): 629-645. https://doi.org/10.1086/590545.
Gopalswamy N. 2017. Extreme solar eruptions and their space weather consequences. In: Extreme events in geospace, Elsevier, pp. 37-63.

Greisen EW, Calabretta MR. 2002. Representations of world coordinates in FITS. $A \& A$ 395: 1061-1075. https://doi.org/ 10.1051/0004-6361:20021326.

Hao Q, Fang C, Cao W, Chen PF. 2015. Statistical analysis of filament features based on the $\mathrm{H} \alpha$ solar images from 1988 to 2013 by computer automated detection method. Astrophys J Suppl Ser 221(2): 33. https://doi.org/10.1088/0067-0049/221/2/33.

Harvey KL, White OR. 1999. Magnetic and radiative variability of solar surface structures. I. Image decomposition and magneticintensity mapping. Astrophys J 515(2): 812-831. https://doi.org/ 10.1086/307035.

Hasan SS, Mallik DCV, Bagare SP, Rajaguru SP. 2010. Solar physics at the Kodaikanal observatory: A historical perspective. Astrophys Space Sci Proc 19: 12-36. https://doi.org/10.1007/9783-642-02859-5_3.

Hathaway DH. 2015. The solar cycle. Liv Rev Sol Phys 12(1): 4. https://doi.org/10.1007/lrsp-2015-4.

Huang Z, Madjarska MS, Koleva K, Doyle JG, Duchlev P, Dechev M, Reardon K. 2014. H $\alpha$ spectroscopy and multiwavelength imaging of a solar flare caused by filament eruption. $A \& A \mathbf{5 6 6}$ : A148. https://doi.org/10.1051/0004-6361/201323097.

Jiang Y, Yang J, Wang H, Ji H, Liu Y, Li H, Li J. 2014. Interaction and merging of two sinistral filaments. Astrophys $J$ 793(1): 14. https://doi.org/10.1088/0004-637X/793/1/14.

Jiang J, Cameron RH, Schüssler M. 2015. The cause of the weak solar cycle 24. Astrophys J Lett 808(1): L28. https://doi.org/ 10.1088/2041-8205/808/1/L28.

Joshi NC, Srivastava AK, Filippov B, Kayshap P, Uddin W, Chandra R, Prasad Choudhary D, Dwivedi BN. 2014. Confined partial filament eruption and its reformation within a stable magnetic flux rope. Astrophys J 787(1): 11. https://doi.org/10.1088/0004-637X/787/1/11.

Kliem B, Török T. 2006. Torus instability. Phys Rev Lett 96(25): 255002. https://doi.org/10.1103/PhysRevLett.96.255002.

Liu R, Liu C, Xu Y, Liu W, Kliem B, Wang H. 2013. Observation of a moreton wave and wave-filament interactions associated with the Renowned X9 Flare on 1990 May 24. Astrophys $J$ 773(2): 166. https://doi.org/10.1088/0004-637X/773/2/166.

Malherbe JM, Dalmasse K. 2019. The new 2018 version of the Meudon Spectroheliograph. Sol Phys. 294(5): 52. https://doi.org/ 10.1007/s11207-019-1441-7.

Malherbe JM, Corbard T, Dalmasse K, Team Meteospace. 2019. Meteospace, a new instrument for solar survey at the Calern observatory. Sol Phys 294(12): 177. https://doi.org/10.1007/ s11207-019-1569-5.

McCauley PI, Su YN, Schanche N, Evans KE, Su C, McKillop S, Reeves KK. 2015. Prominence and filament eruptions observed by the Solar Dynamics observatory: Statistical properties, kinematics, and online catalog. Sol Phys 290(6): 1703-1740. https://doi.org/ 10.1007/s11207-015-0699-7.

McIntosh SW, Leamon RJ, Gurman JB, Olive J-P, Cirtain JW, Hathaway DH, Burkepile J, Miesch M, Markel RS, Sitongia L. 2013. Hemispheric asymmetries of solar photospheric magnetism: Radiative, particulate, and heliospheric impacts. Astrophys $J \mathbf{7 6 5}$ (2): 146. https://doi.org/10.1088/0004-637X/765/2/146.

Moreton GE. 1960. $\mathrm{H} \alpha$ observations of flare-initiated disturbances with velocities $1000 \mathrm{~km} / \mathrm{sec}$. AJ 65: 494. https://doi.org/10.1086/ 108346.

Mouradian Z, Soru-Escaut I. 1993. On solar activity and the solar cycle. A new analysis of the butterfly diagram of sunpsots. $A \& A$ 280(2): 661-665. 
Muhr N, Vršnak B, Temmer M, Veronig AM, Magdalenić J. 2010. Analysis of a global Moreton wave observed on 2003 October 28. Astrophys J 708(2): 1639-1649. https://doi.org/10.1088/0004637X/708/2/1639.

Nagy M, Lemerle A, Labonville F, Petrovay K, Charbonneau P. 2017. The effect of "Rogue" active regions on the solar cycle. Sol Phys 292(11): 167. https://doi.org/10.1007/s11207-017-1194-0.

Narukage N, Ishii TT, Nagata S, UeNo S, Kitai R, Kurokawa H, Akioka M, Shibata K. 2008. Three successive and interacting shock waves generated by a solar flare. Astrophys J Lett 684(1): L45. https://doi.org/10.1086/592108.

Pevtsov AA, Virtanen I, Mursula K, Tlatov A, Bertello L. 2016. Reconstructing solar magnetic fields from historical observations. I. Renormalized Ca K spectroheliograms and pseudo-magnetograms. A\&A 585: A40. https://doi.org/10.1051/0004-6361/201526620.

Pomoell J, Poedts S. 2018. EUHFORIA: European heliospheric forecasting information asset. J Space Weather Space Clim 8: A35. https://doi.org/10.1051/swsc/2018020.

Ribes JC, Nesme-Ribes E. 1993. The solar sunspot cycle in the Maunder minimum AD1645 to AD1715. A\&A 276: 549.

Schmieder B. 2018. Extreme solar storms based on solar magnetic field. J Atmos Sol-Terr Phys 180: 46-51. https://doi.org/10.1016/ j.jastp.2017.07.018.

Schmieder B, Forbes TG, Malherbe JM, Machado ME. 1987. Evidence for gentle chromospheric evaporation during the gradual phase of large solar flares. Astrophys J 317: 956. https://doi.org/ $10.1086 / 165344$.

Schrijver CJ, Beer J, Baltensperger U, Cliver EW, Güdel M, et al. 2012. Estimating the frequency of extremely energetic solar events, based on solar, stellar, lunar, and terrestrial records. $J$ Geophys Res (Space Phys) 117(A8): A08103. https://doi.org/ 10.1029/2012JA017706.

Shibata K, Magara T. 2011. Solar flares: Magnetohydrodynamic processes. Liv Rev Sol Phys 8(1): 6. https://doi.org/10.12942/lrsp2011-6.
Spoerer FWG, Maunder EW. 1890. Prof. Spoerer's researches on Sun-spots. Mon Not R Astron Soc 50: 251. https://doi.org/10.1093/ mnras/50.4.251.

Svalgaard L, Kamide Y. 2013. Asymmetric solar polar field reversals. Astrophys J 763(1): 23. https://doi.org/10.1088/0004637X/763/1/23.

Toriumi S, Schrijver CJ, Harra LK, Hudson H, Nagashima K. 2017. Magnetic properties of solar active regions that govern large solar flares and eruptions. Astrophys $J$ 834(1): 56. https://doi.org/ 10.3847/1538-4357/834/1/56.

Tsiftsi T, De la Luz V. 2018. Extreme value analysis of solar flare events. Space Weather 16(12): 1984-1996. https://doi.org/ 10.1029/2018SW001958.

Ueno S, Shibata K, Ichimoto K, Kitai R, Nagata S, Kimura G, Nakatani Y. 2010. Continuous H-alpha Imaging Network Project (CHAIN) with ground-based solar telescopes for Space Weather Research. African Skies 14: 17.

Veronig AM, Polanec W. 2015. Magnetic reconnection rates and energy release in a confined X-class flare. Sol Phys 290(10): 2923 2942. https://doi.org/10.1007/s11207-015-0789-6.

Wang H, Qiu J, Jing J, Zhang H. 2003. Study of ribbon separation of a flare associated with a Quiescent filament eruption. Astrophys $J$ 593(1): 564-570. https://doi.org/10.1086/376360.

Warmuth A. 2015. Large-scale globally propagating coronal waves. Liv Rev Sol Phys 12(1): 3. https://doi.org/10.1007/lrsp-2015-3.

Webb DF, Gibson SE, Hewins IM, McFadden RH, Emery BA, Malanushenko A, Kuchar TA. 2018. Global solar magnetic field evolution over 4 solar cycles: Use of the McIntosh archive. Front Astron Space Sci 5: 23. https://doi.org/10.3389/fspas. 2018.00023.

Zhang Y, Kitai R, Narukage N, Matsumoto T, Ueno S, Shibata K, Wang J. 2011. Propagation of Moreton waves. Publ Astron Soc Jpn 63: 685. https://doi.org/10.1093/pasj/63.3.685.

Cite this article as: Malherbe J-M, Corbard T \& Dalmasse K 2020. Optical instrumentation for chromospheric monitoring during solar cycle 25 at Paris and Côte d'Azur observatories. J. Space Weather Space Clim. 10, 31. 